To appear in the Journal of Modern Optics

Vol. 00, No. 00, 00 Month 20XX, 1-6

\title{
Statistical properties of the radiation from SASE FEL operating in a post-saturation regime with and without undulator tapering
}

\author{
E.A. Schneidmiller, M.V. Yurkov, DESY, Hamburg, Germany \\ (Received 00 Month 20XX; final version received 00 Month 20XX)
}

\begin{abstract}
We describe statistical and coherence properties of the radiation from x-ray free electron lasers (XFEL) operating in the post-saturation regime. We consider practical case of the SASE3 FEL at the European XFEL. We perform comparison of the main characteristics of the X-ray FEL operating in the postsaturation regime with and without undulator tapering: efficiency, coherence time and degree of transverse coherence.
\end{abstract}

\section{Introduction}

Radiation from Self Amplified Spontaneous Emission Free Electron Laser (SASE FEL) [1, 2] has limited spatial and temporal coherence. This happens due to start-up of the amplification process from the shot noise in the electron beam. The fluctuations of the electron beam density are uncorrelated in time and space, and many radiation modes are excited at the initial stage of amplification. As a rule, ground spatial $\mathrm{TEM}_{00}$ mode with highest gain dominates, and the degree of transverse coherence grows in the exponential amplification stage. Radiation wave slips forward with respect to the electron beam by one wavelength per one undulator period. This relative slippage on the scale of the field gain length gives an estimate for coherence length. Both, the degree of transverse coherence and the coherence time reach maximum value in the end of the exponential regime of amplification, and then degrade visibly in the nonlinear regime. Maximum degree of transverse coherence of about 0.95 is reached for the values of the diffraction parameter about 1 . For large values of the diffraction parameter the degree of transverse coherence falls down due to poor mode selection, i.e. mode degeneration takes place. For small values of the diffraction parameter the degree of transverse coherence falls down due to a poor longitudinal coherence [ 3 [6] .

Radiation from SASE FEL with planar undulator contains visible contribution of the odd harmonics. Parameter range where intensity of higher harmonics is defined mainly by the nonlinear beam bunching in the fundamental harmonic has been intensively studied in refs. [7-16]. Comprehensive studies of the nonlinear harmonic generation have been performed in [16] in the framework of the one-dimensional model. General features of the harmonic radiation have been determined. It was found that the coherence time at the saturation falls inversely proportional to the harmonic number, and relative spectrum bandwidth remains constant with the harmonic number. Comprehensive study of the coherence properties of the odd harmonics in the framework of the three-dimensional model have been performed in [17]. We considered parameter range when the intensity of higher harmonics is mainly defined by the nonlinear harmonics generation mechanism. The case of the optimized XFEL has been considered. Using similarity techniques we derived universal dependencies for the main characteristics of the SASE FEL covering all practical range of optimized X-ray FELs.

Application of the undulator tapering [18] allows to increase the conversion efficiency to rather high values [19 28]. It is used now as a routine procedure at LCLS and SACLA, and is planned for use at SWISS FEL, PAL XFEL, and European XFEL. 29 33]. Simulation studies of the tapered 
SASE FELs have been performed (see [34 37] and references therein).

There are two main reasons why we performed the present study. The first reason is that the SASE3 FEL at the European XFEL, operating at long wavelengths, can not be tuned as optimized FEL [4] due to the limitation on the minimum value of the focusing beta function. Another reason is that in the parameter range of SASE3 FEL the linear mechanism of harmonic generation is essential which results in much higher power of the higher odd harmonic with respect to the case of the nonlinear harmonic generation [34, 39]. In our study we compare coherence properties of the fundamental and the third harmonic for the case of untapered undulator and the undulator with optimized tapering [38]. We show that the brilliance of the fundamental harmonic of the radiation from the SASE FEL with the optimized undulator tapering can be increased by a factor of 3 with respect to untapered case.

\section{General definitions and simulation procedure}

The first order time correlation function $g_{1}\left(t, t^{\prime}\right)$ and the first-order transverse correlation function $\gamma_{1}\left(\vec{r}_{\perp}, \vec{r}_{\perp}, z, t\right)$ are defined as

$$
\begin{aligned}
& g_{1}\left(\vec{r}, t-t^{\prime}\right)=\frac{\left\langle\tilde{E}(\vec{r}, t) \tilde{E}^{*}\left(\vec{r}, t^{\prime}\right)\right\rangle}{\left[\left\langle|\tilde{E}(\vec{r}, t)|^{2}\right\rangle\left\langle\left|\tilde{E}\left(\vec{r}, t^{\prime}\right)\right|^{2}\right\rangle\right]^{1 / 2}}, \\
& \gamma_{1}\left(\vec{r}_{\perp}, \vec{r}_{\perp}, z, t\right)=\frac{\left\langle\tilde{E}\left(\vec{r}_{\perp}, z, t\right) \tilde{E}^{*}\left(\vec{r}_{\perp}, z, t\right)\right\rangle}{\left[\left\langle\left|\tilde{E}\left(\vec{r}_{\perp}, z, t\right)\right|^{2}\right\rangle\left\langle\left|\tilde{E}\left(\vec{r}_{\perp}, z, t\right)\right|^{2}\right\rangle\right]^{1 / 2}},
\end{aligned}
$$

where $\tilde{E}$ is the slowly varying amplitude of the amplified wave. For a stationary random process the time correlation function is the function of arguments $\tau=t-t$, and the first-order transverse correlation function $\gamma_{1}$ does not depend on time. The coherence time and the degree of transverse coherence are defined as:

$$
\begin{aligned}
\tau_{\mathrm{c}} & =\int_{-\infty}^{\infty}\left|g_{1}(\tau)\right|^{2} \mathrm{~d} \tau . \\
\zeta & =\frac{\int\left|\gamma_{1}\left(\vec{r}_{\perp}, \vec{r}_{\perp}\right)\right|^{2} I\left(\vec{r}_{\perp}\right) I\left(\vec{r}_{\perp}\right) \mathrm{d} \vec{r}_{\perp} \mathrm{d} \vec{r}_{\perp}}{\left[\int I\left(\vec{r}_{\perp}\right) \mathrm{d} \vec{r}_{\perp}\right]^{2}}
\end{aligned}
$$

where $I\left(\vec{r}_{\perp}\right)=\left\langle\left|\tilde{E}\left(\vec{r}_{\perp}\right)\right|^{2}\right\rangle$. Peak brilliance is defined as a transversely coherent spectral flux:

$$
B=\frac{\omega \mathrm{d} \dot{N}_{p h}}{\mathrm{~d} \omega} \frac{\zeta}{(\lambda / 2)^{2}}
$$

\section{Results}

Simulations have been performed with the three-dimensional, time-dependent FEL simulation code FAST [4, 17, 40]. In our simulation procedure we trace real number of electrons randomly distributed in the six-dimensional phase space [4, 17]. This allows us to avoid any artificial effects 

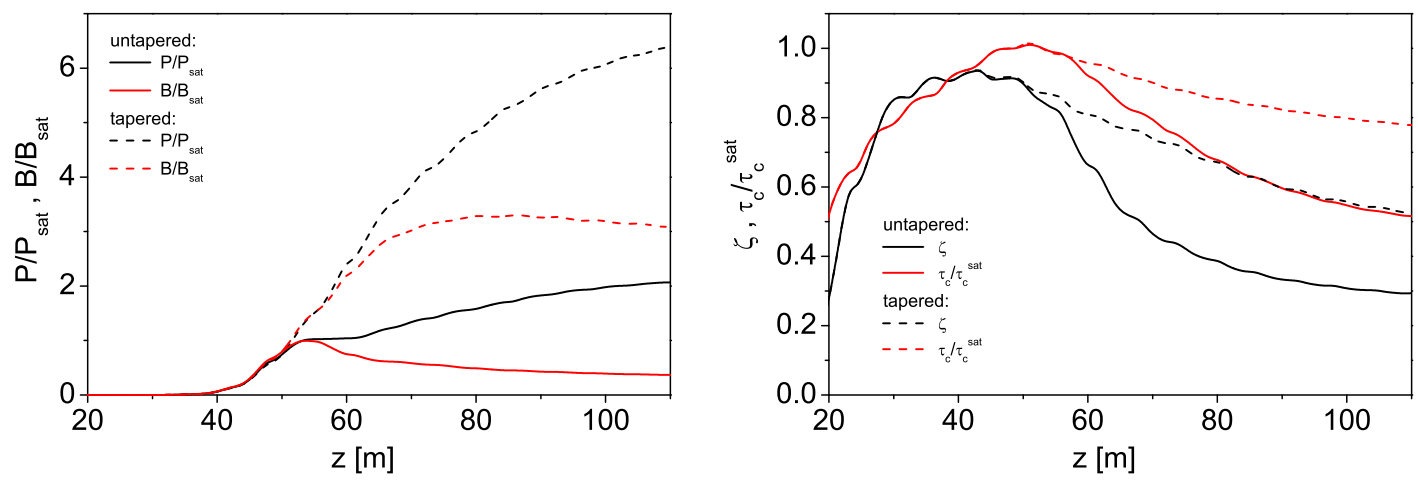

Figure 1. Fundamental harmonic: evolution of the radiation power and brilliance (left plot) and of coherence time and degree of transverse coherence (right plot) along the undulator for untapered (solid curves) and optimized tapered case (dashed curves).

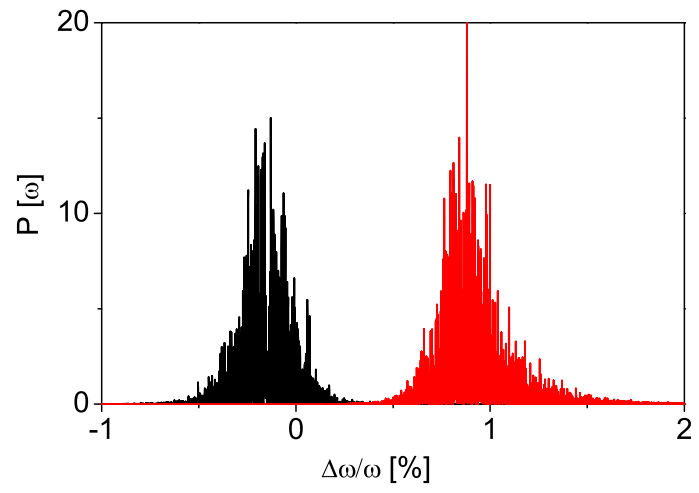

Figure 2. Normalized spectral power of the fundamental harmonic for untapered (black) and tapered (red) case. Output points correspond to the maximum brilliance: $z=53 \mathrm{~m}$ and $z=80 \mathrm{~m}$ for untapered and tapered case, respectively. Spectrum of untapered case is shifted by $1 \%$ to the right-hand side.

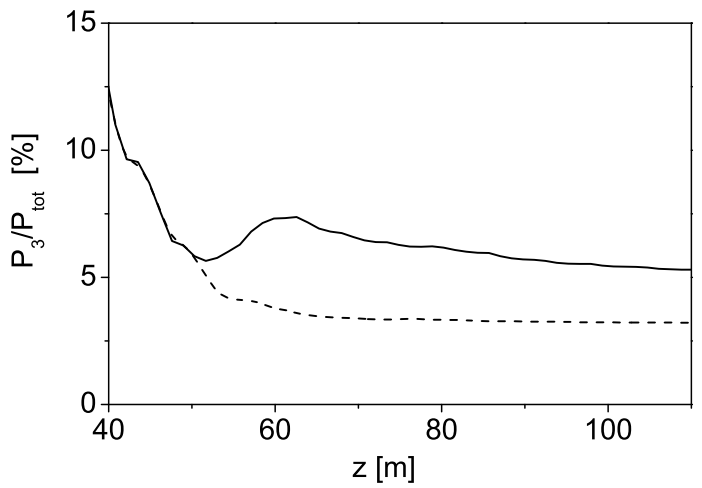

Figure 3. Contribution of the 3rd harmonic radiation power to the total radiation power for untapered (solid curves) and optimized tapered case (dashed curves).

arising from standard procedures of macroparticle loading as we described earlier [4]. Simulations of the FEL process have been performed for the case of a long bunch with the uniform axial profile of the beam current. Output of the simulation code are arrays containing complex values of the radiation field amplitudes. Then we apply statistical analysis and calculate physical values as it has been defined in the previous section. Such a model provides accurate predictions for the coherence properties of the XFEL.

We perform comparative analysis of tapered and untapered case for the parameters of the SASE3 undulator of the European XFEL. Undulator period is $6.8 \mathrm{~cm}$, electron energy is $14 \mathrm{GeV}$, radiation 

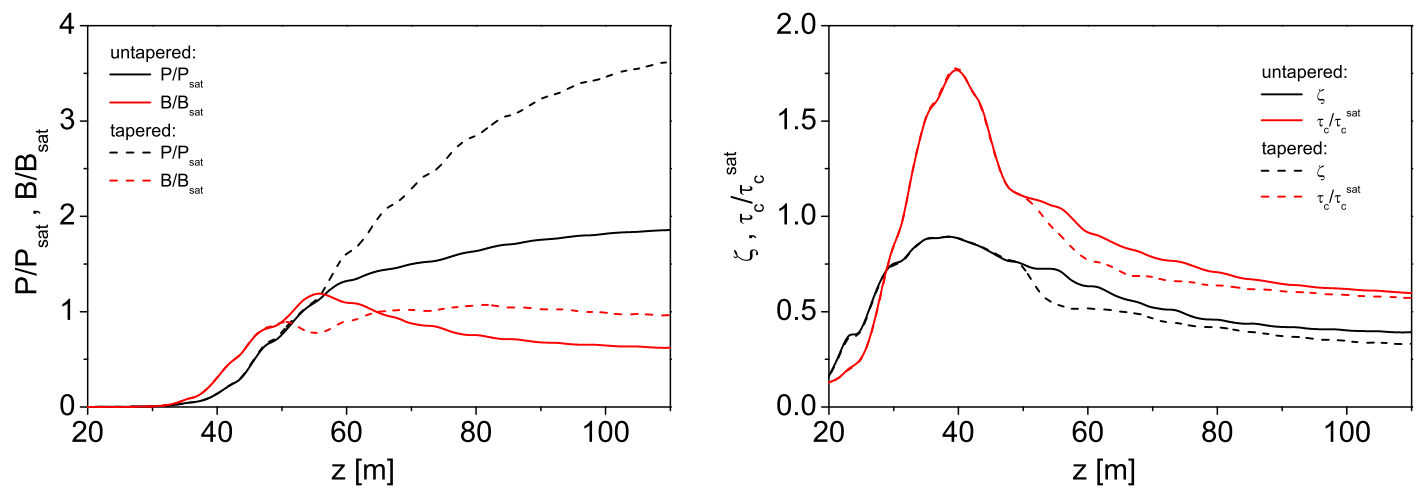

Figure 4. 3rd harmonic: evolution of the radiation power and brilliance (left plot) and of coherence time and degree of transverse coherence (right plot) along the undulator for untapered (solid curves) and optimized tapered case (dashed curves).
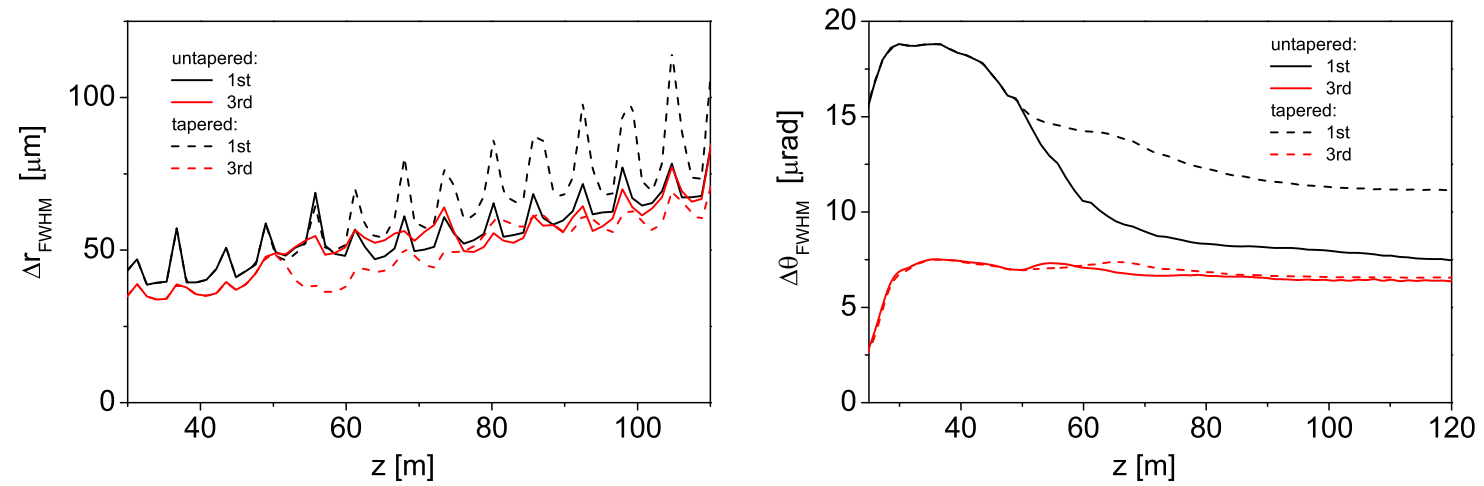

Figure 5. Evolution along the undulator of the FWHM spot size (left plot) and FWHM angular divergence of the radiation in the far zone (right plot) for untapered (solid curves) and optimized tapered case (dashed curves). Black and red colors correspond to the fundamental and the 3rd harmonic, respectively.

wavelength is $1.55 \mathrm{~nm}$. Undulator consists of 21 modules, each is 5 meters long with $1.1 \mathrm{~m}$ long intersections between modules. Parameters of the electron beam correspond to $0.25 \mathrm{nC}$ case of the baseline parameters of the electron beam: emittance $0.6 \mathrm{~mm}$-mrad, rms energy spread $2.5 \mathrm{MeV}$, peak beam current $5 \mathrm{kA} \mathrm{[34]}$. Average focusing beta function is equal to $15 \mathrm{~m}$. The value of the diffraction parameter is $\mathrm{B}=1.1$ which is close to the optimum conditions for reaching the maximum value of the degree of transverse coherence [4]. Two cases were simulated: untapered undulator, and the undulator optimized for maximum FEL efficiency [38].

Plots in Fig. 1 show evolution along the undulator of the radiation power, the degree of transverse coherence, the coherence time, and the brilliance for the fundamental harmonic. For the case of untapered undulator the coherence time and the degree of transverse coherence reach maximum values in the end of the linear regime. Maximum brilliance of the radiation is achieved in the very beginning of the nonlinear regime which is also referred as the saturation point [4]. In the case under study the saturation occurs at the undulator length of $53 \mathrm{~m}$. Parameters of the radiation at the saturation point are: the radiation power is $108 \mathrm{GW}$, the coherence time is $1.2 \mathrm{fs}$, the degree of transverse coherence is 0.86 , and the brilliance of the radiation is equal to $3.8 \times 10^{22}$ photons $/ \mathrm{sec} / \mathrm{mm}^{2} / \mathrm{rad}^{2} / 0.1 \%$ bandwidth. The radiation characteristics plotted in Fig. 1 are normalized to the corresponding values at the saturation point.

General observations for the tapered regime are as follows. Radiation power grows faster in than the untapered tapered case. The coherence time and the degree of transverse coherence degrade, but a bit less intensive than in the untapered case. Brilliance of the radiation for the tapered case saturates at the undulator length of $80 \mathrm{~m}$, and then drops down gradually. Benefit of the tapered case against untapered case in terms of the radiation brilliance is factor of 3 , and it is mainly 
defined by the corresponding increase of the radiation power. Coherence properties of the radiation in the point of the maximum brilliance of the tapered case are worse than those of the untapered SASE FEL in the saturation point: 0.86 to 0.68 for the degree of transverse coherence, and 1 to 0.86 in terms of the coherence time. Figure 2 presents normalized spectral power of the fundamental harmonic for untapered (black) and tapered (red) case. Output points correspond to the maximum brilliance: $z=53 \mathrm{~m}$ and $z=80 \mathrm{~m}$ for untapered and tapered case, respectively. Spectrum of the untapered case is more narrow and does not contain spanning tails.

We already mentioned that parameters of SASE3 FEL are such that we expect significant increase of the radiation power in the higher odd harmonics due to the mechanism of the linear harmonic generation. This happens due to small value of the diffraction parameter and high quality of the electron beam (small emittance and energy spread) [39]. The results of numerical simulations presented in Fig. 3 shows that for the untapered case the contribution of the 3rd harmonic to the total power is about $6 \%$ in the saturation point. Note that the mechanism of the nonlinear harmonic generation results in the value of $2 \%$ only. High value of the 3rd harmonic radiation power should be the subject of concern for the planned user experiments. It can constitute harmful background, or it can be used in the pump-probe experiments. Mechanisms to control the 3rd harmonic radiation are now the subject of dedicated studies [41].

The evolution along the undulator of the radiation power, the degree of transverse coherence, the coherence time, and the brilliance for the 3rd harmonic is shown in Fig. 4. The values are normalized to the corresponding values of the $3 \mathrm{rd}$ harmonic at the saturation point of the fundamental harmonic at the undulator length of $53 \mathrm{~m}$. Normalizing factors are $6.6 \mathrm{GW}$ for the radiation power, $05 \mathrm{fs}$ for the coherence time, and 0.72 for the degree of transverse coherence. There is interesting observation that the brilliance of the radiation of the 3rd harmonic does not differ significantly for the tapered and untapered cases. In the case of the optimized tapered undulator the relative contribution of the 3rd harmonic to the total power is visibly less while the absolute power is higher than for the untapered case. Coherence properties of the 3rd harmonic for untapered case are a bit better than those for the tapered case.

To make our paper complete, we conclude with presenting in Fig. 5 of the evolution along the undulator of the FWHM spot size and the FWHM angular divergence of the radiation in the far zone. The cone of the fundamental harmonic radiation in the far zone is visibly wider for the tapered case. Also, phase front of the radiation is quite different for the tapered and untapered case. This is a hint for careful design of the optical transport system capable effectively handle both, untapered and tapered options.

\section{Summary}

Application of the undulator tapering has evident benefit for SASE3 FEL operating in the wavelength range around $1.6 \mathrm{~nm}$. It is about factor of 6 in the pulse radiation energy with respect to the saturation regime, and factor of 3 with respect to the radiation power at a full length. General feature of tapered regime is that both, spatial and temporal coherence degrade in the nonlinear regime, but more slowly than for untapered case. Peak brilliance is reached in the middle of tapered section, and exceeds by a factor of 3 the value of the peak brilliance in the saturation regime. The degree of transverse coherence at the saturation for untapered case is 0.86 . Degree of transverse coherence for the maximum brilliance of the tapered case is 0.66 . Coherence time falls by $15 \%$. At the exit of the undulator the degree of transverse coherence for the tapered case is 0.6 , and coherence time falls by $20 \%$. Radiation of the 3rd harmonic for both, untapered and tapered cases, exhibit nearly constant brilliance and nearly constant contribution to the total power. Coherence time of the 3rd harmonic for the tapered case approximately scales inversely proportional to harmonic number, as in untapered case. 


\section{References}

[1] Ya.S. Derbenev, A.M. Kondratenko, and E.L. Saldin, Nucl. Instrum. and Methods 193(1982)415.

[2] J.B. Murphy and C. Pellegrini, Nucl. Instrum. and Methods A237(1985)159.

[3] E.L. Saldin, E.A. Schneidmiller, and M.V. Yurkov, Opt. Commun. 186(2000)185.

[4] E.L. Saldin, E.A. Schneidmiller, and M.V. Yurkov, Opt. Commun. 281(2008)1179.

[5] E.L. Saldin, E.A. Schneidmiller, and M.V. Yurkov, Opt. Commun. 281(2008)4727.

[6] E.L. Saldin, E.A. Schneidmiller, and M.V. Yurkov, New J. Phys. 12 (2010) 035010, doi: 10.1088/13672630/12/3/035010.

[7] M. Schmitt and C. Elliot, Phys. Rev. A, 34(1986)6.

[8] R. Bonifacio, L. De Salvo, and P. Pierini, Nucl. Instr. Meth. A 293(1990)627.

[9] W.M. Fawley, Proc. IEEE Part. Acc. Conf., 1995, p.219.

[10] H. Freund, S. Biedron and S. Milton, Nucl. Instr. Meth. A 445(2000)53.

[11] H. Freund, S. Biedron and S. Milton, IEEE J. Quant. Electr. 36(2000)275.

[12] S. Biedron et al., Nucl. Instr. Meth. A 483(2002)94.

[13] S. Biedron et al., Phys. Rev. ST 5(2002)030701.

[14] Z. Huang and K. Kim, Phys. Rev. E, 62(2000)7295.

[15] Z. Huang and K. Kim, Nucl. Instr. Meth. A 475(2001)112.

[16] E.L. Saldin, E.A. Schneidmiller and M.V. Yurkov, Phys. Rev. ST-AB 9(2006)030702

[17] E.A. Schneidmiller and M.V. Yurkov, Proc. 2012 FEL Conference, Nara, Japan, 2012, MOPD08,

[18] N.M. Kroll, P.L. Morton, and M.N. Rosenbluth, IEEE J. Quantum Electron. 17, 1436 (1981).

[19] T.J. Orzechowski et al., Phys. Rev. Lett. 57, 2172 (1986).

[20] R.A. Jong, E.T. Scharlemann, W.M. Fawley Nucl. Instrum. Methods Phys. Res. A272, 99 (1988).

[21] W.M. Fawley, Nucl. Instrum. Methods Phys. Res. A375, 550 (1996).

[22] W.M. Fawley et al., Nucl. Instrum. Methods Phys. Res. A483, 537 (2002).

[23] E.L. Saldin, E.A. Schneidmiller and M.V. Yurkov, Opt. Commun. 95, 141 (1993).

[24] Review by E.T. Scharlemann in Laser Handbook, Volume 6, Free electron lasers, eds. W.B. Colson, C. Peilegrini and A. Renieri (North Holland, Amsterdam, 1991).

[25] E.L. Saldin, E.A. Schneidmiller and M.V. Yurkov, Physics Reports 260, 187 (1995).

[26] E.L. Saldin, E.A. Schneidmiller, M.V. Yurkov, "The Physics of Free Electron Lasers" (Springer-Verlag, Berlin, 1999).

[27] E.A. Schneidmiller, V.F. Vogel, H. Weise and M.V. Yurkov, Journal of Micro/Nanolithogrphy, MEMS, and MOEMS 11(2), 021122 (2012).

[28] Y Hidaka et al., Proceedings of 2011 Particle Accelerator Conference, New York, NY, USA, THP148 (2011).

[29] P. Emma et al., Nature Photonics 4(2010)641.

[30] T. Ishikawa et al., Nature Photonics 6 (2012) 540.

[31] M. Altarelli et al. (Eds.), XFEL: The European X-Ray Free-Electron Laser. Technical Design Report, Preprint DESY 2006-097, DESY, Hamburg, 2006 (see also http://xfel.desy.de).

[32] R. Ganter (Ed.), Swiss FEL Conceptual Design Report, PSI Bericht Nr. 10-04, April 2012.

[33] H.S. Kang, K.W. Kim, I.S. Ko, Current Status of PAL-XFEL Project. Proc. IPAC 2014 Conf., paper THPRO019 (2014).

[34] E.A. Schneidmiller and M.V. Yurkov, Preprint DESY 11-152, Hamburg, 2011.

[35] Y. Jiao et al. Phys. Rev. ST Accel. Beams 15, 050704 (2012).

[36] G. Geloni, V. Kocharyan, and E. Saldin, DESY Report 11-049, 2011.

[37] I. Agapov et al., Proc. 2014 FEL Conference, Basel, Switzerland, 2014, MOP056.

[38] E.A. Schneidmiller and M.V. Yurkov, Proc. 2014 FEL Conference, Basel, Switzerland, 2014, MOP065.

[39] E.A. Schneidmiller and M.V. Yurkov, Phys. Rev. ST-AB 15(2012)080702.

[40] E.L. Saldin, E.A. Schneidmiller, and M.V. Yurkov, Nucl. Instrum. and Methods A 429(1999)233.

[41] E.A. Schneidmiller and M.V. Yurkov, Proc. 2014 FEL Conference, Basel, Switzerland, 2014, MOP068. 